\title{
Análisis de los Modelos Matemáticos Producidos durante la Resolución de Problemas de Fermi*
}

\section{Analysis of Mathematical Models Produced when Solving Fermi Problems}

\begin{abstract}
Resumen
En este trabajo presentamos un estudio en el que analizamos las producciones escritas de estudiantes de 16 años con diferentes niveles de experiencia en modelización al resolver Problemas de Estimación de Grandes Cantidades (PEGC). Estos problemas son un tipo concreto de los Problemas de Fermi y permiten introducir los procesos de modelización en las aulas de Educación Secundaria. Nuestro análisis se soporta en la caracterización de los modelos matemáticos que producen los alumnos, basada en la definición de modelo matemático propuesta por Lesh y Harel. Los resultados muestran que, a través del análisis de las resoluciones de PEGC, se pueden distinguir aspectos diferenciadores entre los modelos producidos por alumnos sin experiencia modelizadora de aquellos producidos por alumnos con experiencia previa, especialmente en los conceptos y lenguajes utilizados para dar forma a los modelos matemáticos construidos.
\end{abstract}

\footnotetext{
* Este trabajo es fruto de una investigación llevada a cabo en el marco de los proyectos de investigación EDU2012-35638 y EDU2013-4683-R que han recibido soporte económico del Ministerio de Economía y Competitividad español y de los Fondos FEDER, así como de la ayuda recibida por parte de la Conselleria de Educació de la Generalitat Valenciana (proyecto GV/2016/129) y la Direcció General de Recerca de la Generalitat de Catalunya (SGR2014-723).

** Doctora por la Universitat Politècnica de València (UPV). Profesora Contratada Doctora interina y secretaria del Dpto. de Didáctica de la Matemática de la Universitat de València (UVEG), Valencia, España. Avda. Tarongers, 4, Facultad de Magisterio, CP. 46022, Valencia, España. E-mail: irene.ferrando@uv.es.

*** Doctor por la Universitat Autonòma de Barcelona (UAB). Profesor Serra Húnter en el Dpt. de Didàctica de la Matemàtica i les Ciències Experimentals de la Universitat Autònoma de Barcelona (UAB), Bellaterra, España. Dirección postal: Campus de la UAB, Edifici G5, 08193, Bellaterra, Barcelona, España. E-mail: lluis.albarracin@uab.cat.

**** Doctor por la por la Universitat Politècnica de València (UPV). Profesor en la Universidad CEU Cardenal Herrera (UCH), Valencia, España. Dirección postal: Facultad de Educación, Avenida Seminario, s/n, 46113, Montcada, Valencia, España.E-mail: gallartcesar@uch.ceu.es.

***** Doctor en CC Físicas por la Universitat de València (UVEG) y Doctor en Matemáticas por la por la Universitat Politècnica de València (UPV). Catedrático en el Dpto. de Matemática Aplicada de la Universitat Politècnica de València (UPV), Valencia, España. Dirección postal: Cami de Vera S/N, 46022, Valencia, España. E-mail: 1mgarcia@mat.upv.es.

****** Doctora por la Universitat Autonòma de Barcelona (UAB). Catedrática en el Dpt. de Didàctica de la Matemàtica i les Ciències Experimentals de la Universitat Autònoma de Barcelona (UAB), Bellaterra, España. Dirección postal: Campus de la UAB, Edifici G5, 08193, Bellaterra, Barcelona, España. E-mail: nuria.gorgorio@uab.cat.
} 
Palabras clave: Tareas de Modelización. Problemas de Fermi. Educación Secundaria.

\begin{abstract}
In this paper, we present an analysis of written productions of 16 year-old students while solving Estimation Problems involving Big Numbers (EPiBN). This kind of problems is a particular type of Fermi problems and allows us to introduce modelling processes in Secondary school classrooms. Our analysis supports on the characterization of mathematical models developed by students based on the model definition of Lesh and Harel. The results show that, through the analysis of EPiBN resolutions, differentiating aspects can be distinguished between the models produced by students without modelling experience of those produced by students with prior experience, especially in the concepts and language used to shape the built mathematical models.
\end{abstract}

Keywords: Modelling tasks. Fermi problems. Secondary School.

\title{
1 Introducción
}

Desde el ICMI 14 (BLUM, 2002) se ha desarrollado un movimiento dentro de la Didáctica de las Matemáticas con la principal idea de llevar a las aulas actividades que muestren la fuerte relación que existe entre las Matemáticas y el mundo que nos rodea. En nuestro entorno educativo, el currículum de Educación Secundaria Obligatoria en España ha incluido, recientemente (Real Decreto 1105/2014, de 26 de diciembre, por el que se establece el currículo básico de la educación secundaria Obligatoria y el Bachillerato, 2015), de forma explícita la práctica de procesos de matematización y modelización, tanto en contextos de la realidad como en contextos propiamente matemáticos.

En estudios previos hemos analizado Problemas de Estimación de Grandes Cantidades (PEGC) que son un tipo concreto de Problemas de Fermi (ALBARRACÍN; GORGORIÓ, 2014). Los problemas que utilizamos presentan situaciones contextualizadas en las que los alumnos deben dar respuestas a interrogantes relacionados con la estimación de grandes cantidades de personas u objetos en un determinado plano, como puede ser la cantidad de personas que asisten a una manifestación o a un concierto multitudinario.

Los PEGC no son habituales en las aulas de Educación Secundaria en España, por lo que los alumnos no poseen métodos o procesos conocidos para resolverlos. Por su naturaleza, los Problemas de Fermi siempre parecen difusos en su planteamiento, dando poca información concreta o pocos aspectos relevantes para enfocar la resolución, pero a partir de un análisis detallado se pueden descomponer en problemas sencillos que permiten llegar a la solución de la pregunta original (EFTHIMIOU; LLEWELLYN, 2007). En estudios previos hemos observado que las producciones de los alumnos, al resolver este tipo de tareas de respuesta abierta, son muy diversas y presentan una gran variedad de estrategias de resolución 
(ALBARRACÍN; GORGORIÓ, 2014). De hecho, los alumnos de Educación Secundaria pueden resolver los PEGC introduciendo elementos de modelización matemática en sus resoluciones (ALBARRACÍN; GORGORIÓ, 2013). Estas características hacen que los PEGC representen una oportunidad para introducir la modelización matemática en las aulas de Educación Secundaria.

En este estudio nos preguntamos si los PEGC pueden representar una categoría de problemas que sirva para caracterizar las diferencias entre alumnos que han realizado tareas de modelización frente alumnos que han seguido una formación tradicional basada en la resolución de problemas de contexto puramente matemático. Para ello presentamos una investigación basada en dos experiencias de aula realizadas en paralelo con alumnos de 16 años que resuelven una misma secuencia de PEGC. Nuestro propósito es analizar los modelos producidos por los alumnos a partir de la definición de modelo matemático de Lesh y Harel (2003) para identificar elementos diferenciadores en las producciones de alumnos con y sin experiencia previa en modelización.

\section{Sobre la modelización matemática}

La resolución de problemas es un ámbito en el que se han realizado un gran número de estudios y avances (LESTER, 1994; SCHOENFELD, 2007) puesto que ha llegado a jugar un papel fundamental en el campo de la Educación matemática en las últimas décadas.

En los últimos años se ha continuado la investigación sobre los procesos de resolución de problemas matemáticos desde diferentes perspectivas y ha aparecido un fuerte interés por el estudio de los procesos de modelización matemática. Refiriendose a los procesos de modelización, Lesh y Harel (2003, p.158) afirman que "the products that problem solvers produce generally involve much more than simply giving brief answers to well formulated questions". En lo que sigue, entendemos que las actividades de modelización matemática son un tipo concreto de tareas de resolución de problemas contextualizados en las cuales es indispensable crear una representación matemática de una realidad o fenómeno, lo que llamamos un modelo.

Así, en nuestro estudio seguiremos la definición de modelo matemático propuesta por Lesh y Harel (2003, p. 159):

Los modelos son sistemas conceptuales que tienden a ser expresados usando una variedad de medios de representación que interactúan entre ellos y que pueden incluir símbolos escritos, lenguaje hablado, gráficos por ordenador, diagramas en 
papel, gráficos o metáforas basadas en la experiencia. Sus propósitos son construir, describir o explicar otro(s) sistema(s).

Los modelos incluyen tanto: (a) un sistema conceptual para describir o explicar los objetos matemáticos relevantes, así como las relaciones, acciones, patrones y regularidades que se atribuyen a la situación de resolución de problemas; y (b) los procedimientos que los acompañan para generar construcciones útiles, manipulaciones o predicciones para el logro de objetivos claramente reconocidos.

A partir de esta definición, entendemos que la elaboración y creación de modelos matemáticos destinados a describir o representar de forma abstracta un determinado fenómeno o realidad es una tarea compleja, en la que intervienen diversos procesos y factores que condicionan la forma del modelo final construido. Por ello consideramos que en un modelo matemático tienen cabida diferentes elementos que le dan forma, como pueden ser los conceptos matemáticos implicados, las representaciones simbólicas de la realidad o esquemas utilizados, así como los procedimientos matemáticos o de otro tipo asociados a su uso. Además, tal y como muestran algunos trabajos, entre los que destacamos el de Silva Campos y Loiola Araújo (2015), las tareas de modelización dan la oportunidad a los alumnos desarrollar, a través de posibilidades de acción, conceptos y procedimientos matemáticos.

\section{Estimación y problemas de Fermi}

Una estimación es un juicio del valor del resultado de una operación numérica o de la medida de una cantidad, en función de las circunstancias y necesidades de la persona que emite ese juicio (SEGOVIA et al., 1989). La estimación aparece en muchas situaciones de la vida cotidiana en las que se requiere una respuesta rápida y ágil a una pregunta. Una buena muestra del tipo de situaciones en las que un modelo matemático es la base para realizar una estimación de una cantidad son los denominados problemas de Fermi, propuestos originalmente por Enricco Fermi (1901-1954). Para Sriraman y Knott (2009) los problemas de Fermi son problemas de estimación con objetivos didácticos relacionados con la elaboración de suposiciones razonadas (educated guesses).

La definición de problema de Fermi que ofrece Ärlebäck (2009, p.331) es la siguiente: "Problemas abiertos, no-estándar que requieren que los estudiantes hagan suposiciones acerca de la situación del problema y estimaciones sobre las cantidades pertinentes antes de iniciar, a menudo, una serie de cálculos sencillos".

El problema ¿cuántos afinadores de piano hay en Chicago? es el que se utiliza para ejemplificar la naturaleza de los problemas de Fermi. La resolución completa del problema, 
que depende de factores que deben ser estimados, como la cantidad de familias que viven en Chicago, la proporción de estas que poseen un piano o el número de horas necesario para afinarlo, se puede consultar en García-Navarro (2013). Efthimiou y Llewellyn (2007) caracterizan los problemas de Fermi a partir de su particular formulación, ya que siempre parecen difusos en su planteamiento y ofrecen poca información concreta al resolutor para enfocar el proceso de resolución. Es a partir de un análisis detallado de la situación presentada en el enunciado que se puede descomponer en problemas más sencillos, que permitan llegar a la solución de la pregunta original a partir de conjeturas razonadas (CARLSON, 1997).

Peter-Koop (2009) ha usado los problemas de Fermi con alumnos de los cursos superiores de Primaria para analizar sus estrategias de resolución. A partir de sus investigaciones concluye que a) los alumnos resuelven los problemas de formas muy variadas, b) desarrollan nuevos conocimientos matemáticos en sus resoluciones y c) en la resolución se intuyen procesos de resolución multicíclicos que se pueden relacionar con la modelización. En Albarracín y Gorgorió (2013, 2014) se analizan las estrategias de resolución de alumnos de Educación Secundaria a diversos problemas de Fermi (PEGC, en este caso) y se observan, también, un gran número de estrategias posibles y diversos elementos de modelización para elaborar las respuestas.

Ärlebäck (2011) afirma que el trabajo con problemas de Fermi puede ser útil para introducir la modelización en las aulas por los siguientes motivos: 1) son accesibles para alumnos de diferentes etapas educativas y no dependen de un tipo concreto de conocimiento matemático previo; 2) fuerzan a los alumnos a especificar la estructura de la información relevante; 3) requieren que el alumno elabore una estrategia de resolución específica para el contexto; 4) al no ofrecer datos numéricos, los alumnos deben estimar diversas cantidades por ellos mismos; y 5) promueven la discusión entre los alumnos.

Por su naturaleza, los problemas de Fermi permiten estrategias de resolución más sencillas que las tareas abiertas de modelización, aunque en ambos casos los alumnos ponen en juego varias competencias con interrelaciones complejas. De esta forma, se observa que existe una fuerte conexión entre los procesos de resolución de los problemas de Fermi y el trabajo desarrollado durante el ciclo de modelización para la elaboración de un modelo matemático (ÄRLEBÄCK, 2009; BORROMEO-FERRI, 2006) sobre todo en las primeras experiencias de los alumnos. Por este motivo consideramos que los problemas de Fermi, y los PEGC en concreto, representan una oportunidad para investigar la competencia modelizadora 
de los alumnos, tanto si han seguido una enseñanza tradicional o han trabajado de forma específica la resolución de tareas de modelización.

\section{Objetivos del estudio}

En este artículo estudiamos el trabajo de aula de alumnos de $4^{\circ}$ de Educación Secundaria Obligatoria (16 años) con una secuencia de Problemas de estimación de grandes cantidades. La secuencia de problemas utilizada ha sido diseñada con la intención de proporcionar a los alumnos situaciones, inicialmente próximas a su realidad y progresivamente más distantes, que les permitan desarrollar sus propios modelos matemáticos y adaptarlos a nuevas situaciones. En este proceso, se espera que los alumnos introduzcan diferentes niveles de complejidad en sus resoluciones produciendo modelos adaptados a cada problema.

De esta forma, los objetivos de nuestro estudio son los siguientes:

1. Caracterizar los modelos matemáticos producidos por alumnos de $4^{\mathrm{a}}$ de $\mathrm{ESO}$ (16 años) durante una secuencia de PEGC a partir de la definición de modelo matemático de Harel y Lesh (2003).

2. Identificar los elementos diferenciadores entre los modelos producidos por alumnos con experiencia previa en actividades de modelización y los modelos producidos por los que no la tienen.

\section{Metodología: diseño del estudio y recogida de datos}

El estudio que presentamos utiliza los datos, recogidos en dos centros diferentes, obtenidos a partir de las resoluciones elaboradas por alumnos de 16 años en una secuencia de PEGC. Partimos del conocimiento previo de que los PEGC permiten el trabajo en el aula relacionado con la generación y uso de modelos matemáticos en su proceso de resolución por parte de los alumnos de Educación Secundaria (ALBARRRACÍN; GORGORIÓ, 2013). Dado que los PEGC no son habituales en las aulas en España, los alumnos no conocen métodos específicos para resolverlos y deben generarlos por ellos mismos a partir de su bagaje como resolutores de problemas. Al tratar los problemas sobre grandes cantidades no resulta factible realizar aproximaciones simplistas a la solución, como utilizar recuentos exhaustivos de objetos o personas, con lo que este tipo de problemas provoca la necesidad en los alumnos 
generar estrategias matemáticamente ricas que incluyan elementos matemáticos para representar la realidad estudiada (ALBARRRACÍN; GORGORIÓ, 2014).

Para orientar el diseño de la secuencia de problemas utilizada se han considerado los aspectos que definen las actividades que promueven la modelización o modelling-eliciting activities (LESH et al., 2000). En concreto, hemos seguido las recomendaciones de Wessels (2014), tratando que las actividades propuestas sean complejas, alejadas en todo caso de los problemas convencionales que van asociados a procedimientos de resolución definidos y que incluyan diferentes contextos reales.

De esta forma, la secuencia de PEGC utilizada es una secuencia de problemas de Fermi que tratan el mismo tipo de problemática desde el sentido estrictamente matemático, ya que todos los problemas piden a los alumnos una estimación del número de personas u objetos que se pueden colocar en una determinada superficie en un determinado contexto. El diseño de la actividad se basa en proponer a los alumnos un primer problema que permite el trabajo de campo en el propio centro educativo, con lo que los alumnos pueden desarrollar métodos de resolución que pueden ejecutar. Después de una sesión en la que los alumnos comparten sus métodos de resolución para este primer problema, se plantea a los alumnos la resolución de un grupo de problemas que no pueden resolverse experimentalmente en un entorno accesible, con lo que se genera la necesidad de transferir las estrategias de resolución del primer problema a otros contextos. Durante estas sesiones, el profesor actúa como guía, marcando los tiempos de trabajo y gestionando las puestas en común de los resultados obtenidos, pero sin intervenir directamente proporcionando directrices o contenidos concretos a los alumnos.

Los enunciados de los problemas que forman la secuencia didáctica, y que presentan variaciones para atender a la localización de los contextos planteados, son los siguientes:

- Problema A: ¿Cuánta gente cabe en el patio del instituto?

- Problema B1: ¿Cuánta gente cabe en el Palau St. Jordi ${ }^{1} /$ Pabellón la Font de Sant Lluís ${ }^{2}$ en un concierto?

- Problema B2: ¿Cuánta gente cabe en la plaza del ayuntamiento de tu ciudad/Plaça de Sant Jaume ${ }^{3}$ en una manifestación?

- Problema B3: ¿Cuántos árboles hay en Central Park?

\footnotetext{
${ }^{1}$ El Palau St. Jordi es un pabellón deportivo ubicado en Barcelona.

${ }^{2}$ El Pabellón Municipal de la Font de Sant Lluís es un pabellón deportivo ubicado en Valencia.

${ }^{3}$ Las dos plazas utilizadas en esta pregunta tienen caraterísticas similares y son próximas a los respectivos centros escolares de los alumnos.
} 
En primer lugar, los alumnos resuelven el problema A, comparten sus métodos y resultados en una puesta en común y continúan el trabajo con la resolución de los problemas $\mathrm{B}$, permitiéndoles acceso a Internet para hacer consultas necesarias.

La experiencia que presentamos se ha realizado con dos grupos de alumnos, uno de 24 alumnos de $4^{\circ}$ de ESO (16 años) que forman 7 equipos de trabajo, de un centro de la provincia de Valencia (grupo E1 en adelante) y otro de 22 alumnos del mismo nivel educativo de un centro de Barcelona (grupo E2 en adelante) que forman 6 equipos de trabajo. Estos últimos no tenían experiencia previa trabajando en actividades de modelización.

Los alumnos del grupo E1 han participado previamente en actividades del mismo tipo a las presentadas en Gallart, Ferrando y García-Raffi (2015a, 2015b). La experiencia en modelización de estos alumnos se desarrolló a lo largo de cinco sesiones de aula, incluyendo una sesión de introducción inicial y una última sesión de comunicación final de sus producciones. Los alumnos trabajaron en equipos, de forma independiente y autónoma, una de las tareas de modelización propuestas. Estas tareas eran abiertas, situadas en contextos reales y cercanos al alumno, y en las que era necesario transitar varias veces por el ciclo de modelización para su resolución. Estos alumnos también comunicaron sus métodos y resultados utilizando informes y presentaciones orales.

De esta forma, los datos de los que disponemos para este estudio son los siguientes:

- Los $7($ E1) + 6 (E2) informes de resolución grupal de los problemas A, B1 y B2.

- $\quad$ Los $7($ E1) + 5 (E2) informes de resolución grupal del problema B3.

\section{Proceso de análisis}

Para analizar los modelos matemáticos generados por los alumnos, durante la secuencia de PEGC, proponemos caracterizar cualitativamente los aspectos esenciales que los definen y que nos proporcionan una visión objetiva de la complejidad con la que estos modelos representan la situación estudiada. Siguiendo a Lesh y Harel (2003), consideramos que un modelo matemático incluye conceptos y procedimientos de forma interrelacionada. En la literatura aparecen numerosas referencias relativas al conocimiento conceptual y procedimental. Los orígenes de ambos términos remiten al trabajo seminal de Hiebert y Lefevre (1986, p.3-4), en él se define el conocimiento conceptual como sigue:

El conocimiento que es rico en relaciones. Se puede considerar como una estructura conectada de conocimiento, una red en la que las relaciones son tan prominentes como las piezas discretas de información. Las relaciones impregnan los hechos y 
proposiciones individuales, de manera que todos los elementos de información están vinculados a alguna red.

Para definir el conocimiento procedimental, Hiebert y Lefevre (1986, p. 7-8) distinguen dos tipos:

\begin{abstract}
Un tipo de conocimiento procedimental es la familiaridad con los símbolos individuales del sistema y con las convenciones sintácticas para configuraciones aceptables de símbolos. El segundo tipo de conocimiento procedimental consiste en reglas o procedimientos para la resolución de problemas matemáticos. Muchos de los procedimientos que los alumnos poseen probablemente son cadenas de recetas para la manipulación de símbolos.
\end{abstract}

Podemos observar que el conocimiento procedimental incluye el conocimiento del uso de los símbolos matemáticos, pero en nuestra propuesta incluimos, también, otras formas de comunicar los resultados. En concreto, consideramos que en el momento de comunicar el modelo producido intervienen símbolos, gráficos, diagramas, lenguaje escrito o algebraico que pueden complementar la carga conceptual del modelo.

Hemos resumido, en el Cuadro 1, los elementos para la caracterización de los modelos matemáticos generados por los alumnos.

\begin{tabular}{|l|l|}
\hline $\begin{array}{l}\text { Sistemas } \\
\text { conceptuales }\end{array}$ & $\begin{array}{l}\text { Objetos matemáticos } \\
\text { Relaciones entre los diferentes objetos matemáticos } \\
\text { Patrones o regularidades en los datos }\end{array}$ \\
\hline Procedimientos & $\begin{array}{l}\text { Algoritmos de cálculo } \\
\text { Medidas } \\
\text { Uso de procedimientos algébricos o funcionales }\end{array}$ \\
\hline Lenguajes & $\begin{array}{l}\text { Lenguaje simbólico } \\
\text { Lenguaje escrito } \\
\text { Lenguaje gráfico, realización de esquemas o diagramas }\end{array}$ \\
\hline
\end{tabular}

Cuadro 1 - Elementos de caracterización de modelos matemáticos Fuente: Cuadro realizado por los autores del trabajo

A partir de este constructo, hemos analizado los modelos propuestos por los alumnos de los dos grupos experimentales al enfrentarse al problema A y a todos los problemas B. A continuación, mostramos los resultados del análisis de las producciones de los alumnos para cada uno de los elementos de caracterización de los modelos matemáticos propuestos.

\title{
7 Resultados: sistemas conceptuales
}

Si nos centramos en el análisis de los sistemas conceptuales utilizados en las resoluciones de los cuatro problemas, hemos detectado conceptos que fundamentan la solución presentada por los alumnos a dos niveles. Por una parte, aparecen conceptos que sustentan la concreción de los modelos producidos para representar el fenómeno estudiado y, 
por otra parte, hemos detectado elementos que matizan el uso de esos conceptos y añaden complejidad a los modelos propuestos.

Respecto a los conceptos fundamentales detectados, los modelos producidos se basan esencialmente en dos de ellos. El primero es la noción de densidad de población, entendida como el número de elementos ubicados en un espacio determinado y expresado en la cantidad de elementos por unidad de superficie. El segundo lo denominamos uso de una unidad base, que entendemos que está relacionado con la estrategia de estimación de medidas conocida como iteración de la unidad (HILDRETH, 1983) que se basa en trabajar con un elemento que actúa como unidad de medida, una vez determinada la superficie que ocupa.

También se ha identificado otro concepto que fundamenta los modelos elaborados por uno de los equipos. En este caso, nos encontramos con el uso de una cuadrícula como imagen mental de la disposición de elementos en el plano, que se acompaña del uso de la regla del producto para realizar los cálculos pertinentes. Finalmente, una estrategia detectada que no soporta un modelo matemático el uso de información proveniente de una fuente externa para obtener resultados numéricos - en concreto, hallan la información en una web-. Las Tablas 1 y 2 muestran los resultados obtenidos en el análisis de sistemas conceptuales para cada uno de los grupos.

Tabla 1 - Sistemas conceptuales grupo E1

\begin{tabular}{llllll}
\hline Problema & Densidad & Unidad base & Cuadrícula & Fuente externa & Total \\
\hline A & 6 & 1 & 0 & 0 & 7 \\
B1 & 5 & 1 & 0 & 1 & 7 \\
B2 & 7 & 0 & 0 & 0 & 7 \\
B3 & 3 & 4 & 0 & 0 & 7 \\
\hline \multicolumn{5}{r}{}
\end{tabular}

Tabla 2 - Sistemas conceptuales grupo E2

\begin{tabular}{llllll}
\hline Problema & Densidad & Unidad base & Cuadrícula & Fuente externa & Total \\
\hline A & 3 & 2 & 1 & 0 & 6 \\
B1 & 5 & 1 & 0 & 0 & 6 \\
B2 & 5 & 1 & 0 & 0 & 6 \\
B3 & 3 & 2 & 0 & 0 & 5 \\
\hline \multicolumn{5}{r}{ Fuente: tabla realizada por los autores del trabajo }
\end{tabular}

A partir de estos resultados, observamos que los alumnos del grupo E1 al enfrentarse a los problemas A, B1 y B2, donde deben calcular la cantidad de personas que caben en el patio/pabellón/plaza, utilizan mayoritariamente la noción de densidad, averiguando el número de personas que caben en un espacio determinado, mientras que esos mismos alumnos no usan el mismo concepto en el problema B3 al tratar de estimar la cantidad de árboles. Al preguntar directamente a los alumnos sobre este hecho, corroboramos que los alumnos del grupo E1 diferencian sus procesos de resolución según si tienen que realizar recuentos de 
personas o de árboles debido a su diferente forma. Respecto a los modelos basados en el concepto de densidad, no todos toman el metro cuadrado como unidad de referencia. Encontramos un equipo del grupo E1 que la utiliza a través de unidades no estandarizadas: toman las baldosas del patio como unidad de área y suponen que en una baldosa caben 6 personas (véase Figura 1).

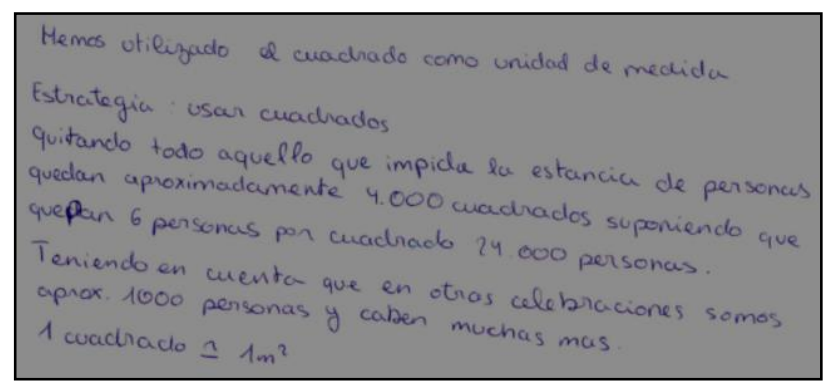

Figura 1 - Resolución del problema A realizada por un equipo del grupo E1 Fuente: producción alumnos de uno de los grupos experimentales del trabajo

Observamos también, en los datos recogidos, aspectos que complementan los conceptos principales en las resoluciones de los problemas. En concreto, observamos resoluciones que señalan espacios hábiles o no, es decir, tienen en cuenta que no todo el espacio disponible en el patio, el pabellón o la plaza puede ser utilizado para albergar asistentes al concierto (véase Figura 1) o, en el caso del problema B3, que no todo el Central Park está lleno de árboles. Otro elemento de complejidad observado y que se relaciona directamente con los modelos basados en la densidad de población es la consideración de diferentes valores de densidad en diferentes zonas del espacio estudiado (véase Figura 2). En la Tabla 3 mostramos el número de equipos de trabajo que han considerado, para cada uno de los problemas, estos elementos de complejidad considerados.

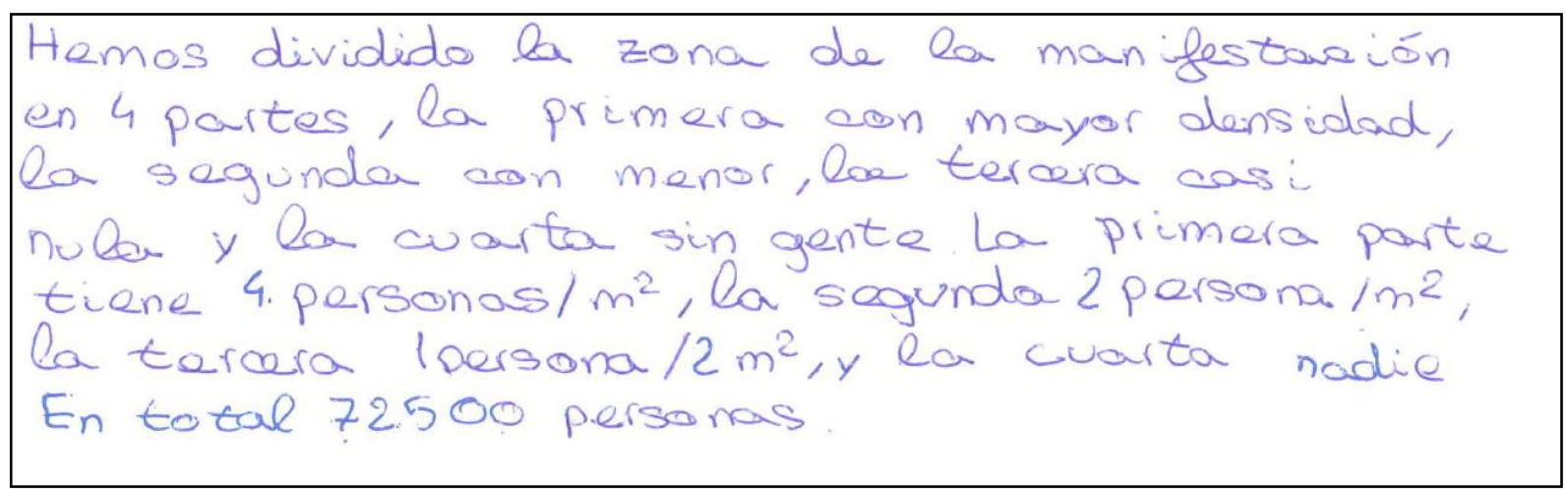

Figura 2 - Resolución del problema B2 realizada por un equipo del grupo E Fuente: producción alumnos de uno de los grupos experimentales del trabajo

Tabla 3 - Elementos de complejidad detectados

\begin{tabular}{|l|l|l|}
\hline Problema & $\begin{array}{l}\text { Espacio hábil/no } \\
\text { hábil }\end{array}$ & $\begin{array}{l}\text { Diferenciación } \\
\text { de zonas }\end{array}$ \\
\hline
\end{tabular}




\begin{tabular}{|l|l|l|l|l|}
\hline & E1 & E2 & E1 & E2 \\
\hline A & 7 & 2 & 0 & 0 \\
\hline B1 & 2 & 1 & 6 & 5 \\
\hline B2 & 3 & 1 & 1 & 0 \\
\hline B3 & 5 & 1 & 0 & 0 \\
\hline Total & 17 & 5 & 7 & 5 \\
\hline
\end{tabular}

Fuente: tabla realizada por los autores del trabajo

El análisis de los conceptos y elementos de complejidad que fundamentan los modelos nos permite identificar aspectos diferenciadores entre las resoluciones de ambos grupos. Las resoluciones del grupo E1 contrastan, significativamente, con las del grupo E2, ya que éstos usaron menos la noción de densidad en los diferentes problemas, pero esta diferencia se acentúa en las resoluciones del problema A, que es el primero de esta tipología que trataban de resolver los alumnos del grupo E2, que carecían de experiencia previa. Otra diferencia significativa es la mayor incidencia de resoluciones que consideran zonas con diferentes densidades en las resoluciones de los alumnos del grupo E1. Esta consideración se encuentra sólo de forma puntual entre los alumnos del grupo E2. Si consideramos la inclusión de espacios hábiles o inhábiles en las resoluciones, observamos que solo 5 de las resoluciones de los equipos del grupo E2 las consideran, por 17 de las resoluciones realizadas por los alumnos del grupo E1. Aunque estos elementos de complejidad no son exclusivos de los alumnos que ya han trabajado en tareas de modelización, se puede observar que éstos los introducen con mayor frecuencia, con lo que desarrollan modelos matemáticos más ajustados a la situación inicial.

\section{Resultados: procedimientos}

Respecto a los procedimientos utilizados en las resoluciones, identificamos tres procedimientos que pueden o no aparecer simultáneamente en la resolución de un problema. En particular, describimos como procedimientos los procesos asociados a las mediciones, a la aproximación de formas geométricas irregulares a partir de la descomposición en figuras simples y a los cálculos aritméticos.

Por la propia configuración de la secuencia de problemas utilizada, encontramos dos tipos de mediciones: mediciones sobre el terreno y mediciones realizadas mediante las herramientas de Google Maps o Google Earth. Las mediciones in situ aparecen solo en la resolución del problema A - pues era el único que se trabajaba también sobre el terreno - pero es interesante apreciar las diferencias entre las resoluciones de ambos grupos: todos los 
equipos del grupo E1 realizan sus mediciones con cinta métrica, salvo uno de ellos que averigua el área a partir del recuento de baldosas (Figura 1). Sin embargo, en el trabajo de los equipos del grupo E2 encontramos dos ejemplos de procedimientos alternativos a las medidas convencionales: uno de los equipos obvia las mediciones y el cálculo del área ya que distribuyen a los asistentes en la vertical y en la horizontal del patio para determinar el número de posibles asistentes a partir de la regla del producto (Figura 3) y otro propone aproximar el área del patio mediante unidades de medida no convencionales, usando sus pasos para estimar la longitud y amplitud del patio.

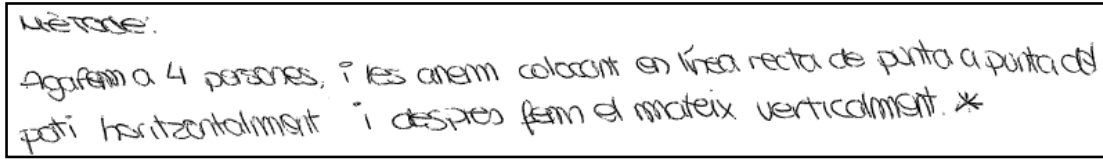

Figura 3 - Resolución del problema A realizada por un equipo del grupo E2 4

Fuente: producción alumnos de uno de los grupos experimentales del trabajo

Otro procedimiento en el que interviene la medición es el uso de la media aritmética para obtener una aproximación de una cantidad variable por muestreo. La encontramos, por ejemplo, en una resolución del problema B3 en el que los alumnos midieron, a través de herramientas digitales, distintas distancias entre árboles de Central Park y establecieron, a través de la media aritmética de las medidas tomadas, un valor medio para la distancia entre ellos (véase Figura 5).

Los procedimientos asociados al cálculo de áreas para los problemas B se realizan en todos los casos a partir de mediciones realizadas con las herramientas de Google Earth. No hay diferencias significativas respecto al uso de estas herramientas entre los alumnos de los dos grupos. Sin embargo, para el problema B2 planteado a los alumnos del grupo E1, observamos un proceso más complejo, como es la aproximación de una forma irregular por una composición de figuras simples. Este hecho, como se puede observar en la Figura 4, es debido a la forma de la Plaça Sant Jaume ya que no es rectangular como la que aparece en el problema equivalente para los alumnos de grupo E2.

\footnotetext{
${ }^{4}$ Traducción realizada por los autores:

Método: Cogeremos a 4 personas y las iremos colocando en línea recta de punta a punta del patio, horizontalmente y después haremos lo mismo verticalmente.

Traducción realizada por los autores.
} 


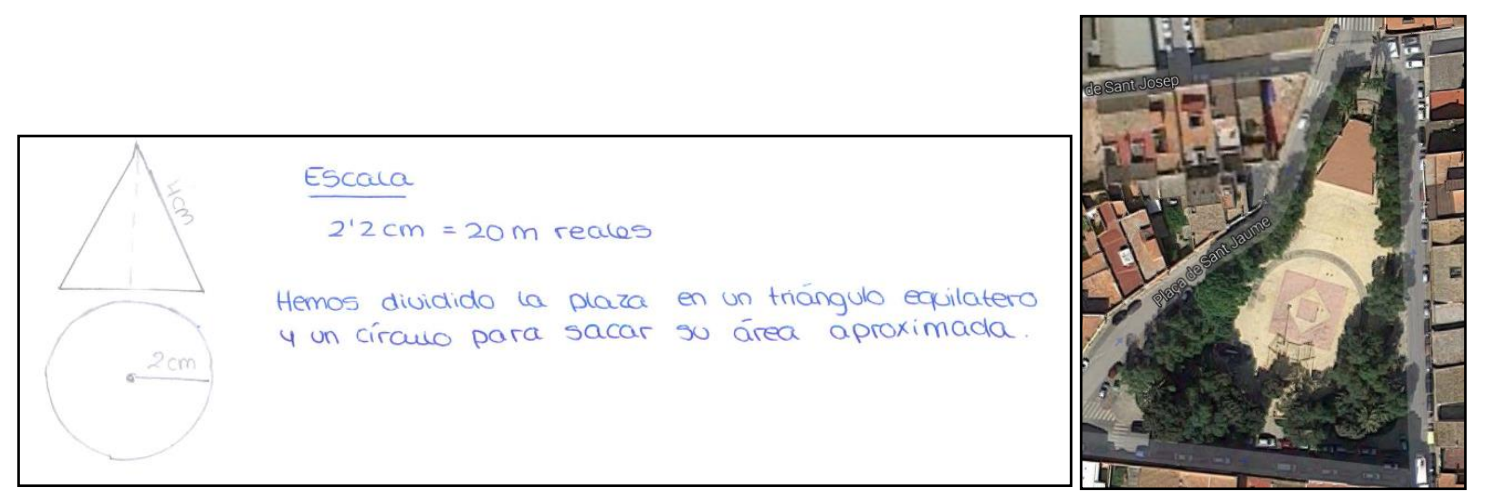

Figura 4 - Resolución del problema B2 realizada por un equipo del grupo E1 e imagen de la Plaça Sant Jaume Fuente: producción alumnos de uno de los grupos experimentales del trabajo, imagen extraída de Google Earth

Algunos de los alumnos que razonan a través de la iteración de la unidad usan procedimientos relacionados con la aproximación de una forma irregular por una figura simple para obtener el área de esta unidad base. Encontramos este procedimiento, por ejemplo, en la resolución del problema B3, donde un equipo alumnos aproxima el área ocupada por un árbol a través del área del cuadrado menor que lo contiene, otros idealizan el espacio ocupado por un árbol como una circunferencia (véase Figura 5).

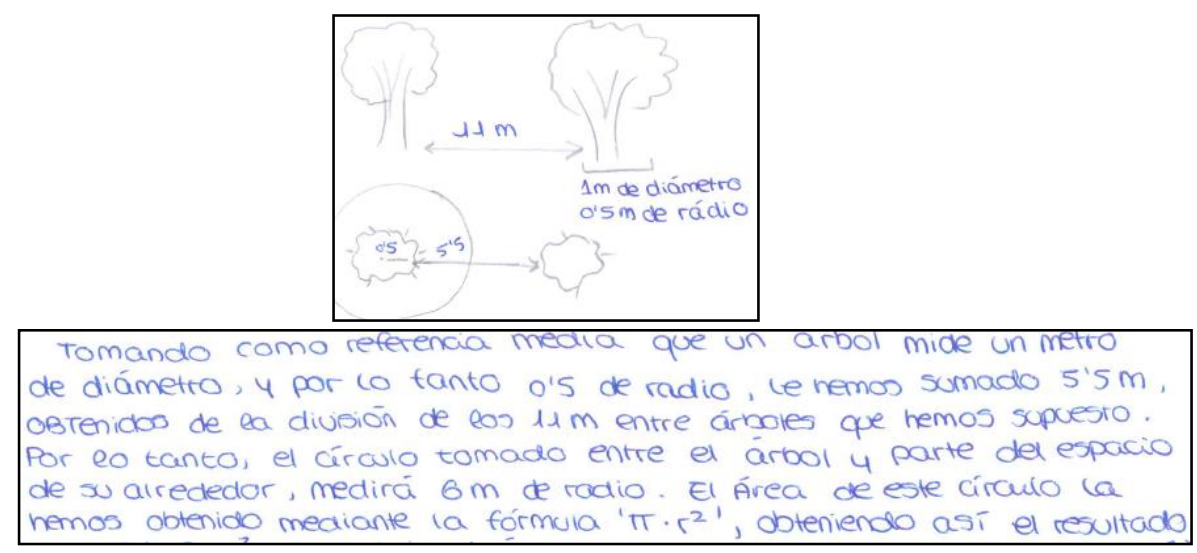

Figura 5 - Resolución del problema B3 realizada por un equipo del grupo E1 Fuente: producción alumnos de uno de los grupos experimentales del trabajo

Por otra parte, hemos encontrado diferentes procedimientos aritméticos utilizados por equipos que razonan a través de sistemas conceptuales equivalentes. En concreto, los alumnos que razonan a partir de la densidad hallan el número de personas (problemas A, B1, B2) o de árboles (problema B3) multiplicando ésta por el área útil. Otro procedimiento para hallar el número total de elementos a partir de la densidad es el uso del razonamiento a partir de la equivalencia de fracciones u otros métodos de cálculo de proporcionalidades como muestra la Figura 6. 


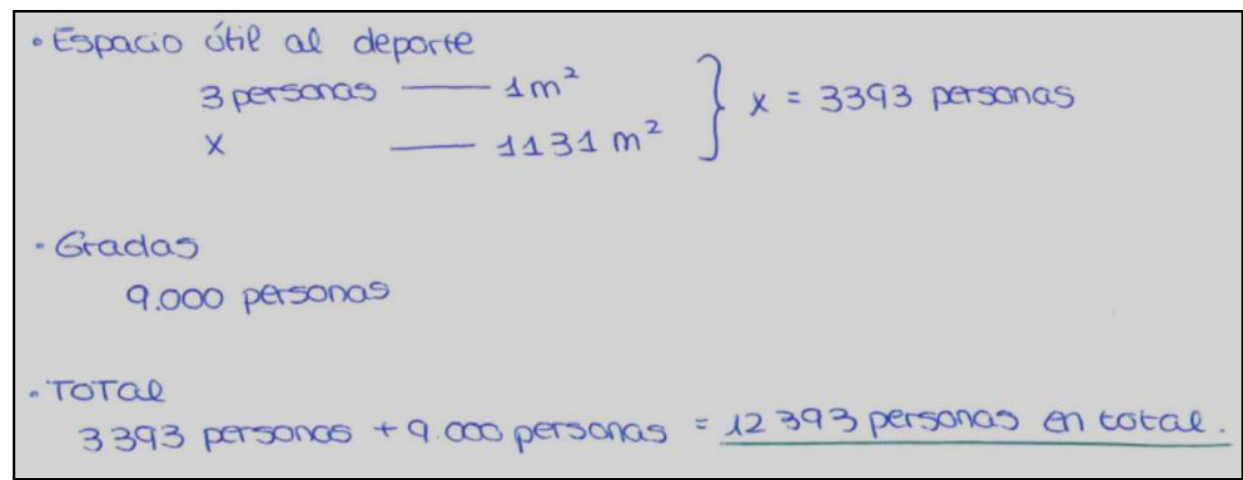

Figura 6 - Resolución al problema B1 realizada por un equipo del grupo E1 Fuente: producción alumnos de uno de los grupos experimentales del trabajo

Los alumnos que utilizan sistemas conceptuales relativos a iteraciones de la unidad razonan a partir de área ocupada por una persona o por un árbol y obtienen la cantidad total dividiendo el área del recinto por el área de la unidad.

En general, observamos una gran diversidad de procedimientos, pero no hemos encontrado evidencias de usos diferenciadores de estos procedimientos en las resoluciones de los dos grupos (salvo en el caso del problema B2 previamente comentado).

\section{Resultados: lenguajes}

Respecto a los lenguajes utilizados, distinguimos entre los gráficos y el texto que compone el informe de resolución de los problemas junto a los cálculos que lo acompañan.

Entre los gráficos que acompañan las resoluciones escritas hemos observado usos distintos. Por una parte, hemos encontrado que, para el problema A, 5 del total de grupos de trabajo utilizan gráficos que sirven para representar la información recogida en el patio, como se muestra en la Figura 7. 


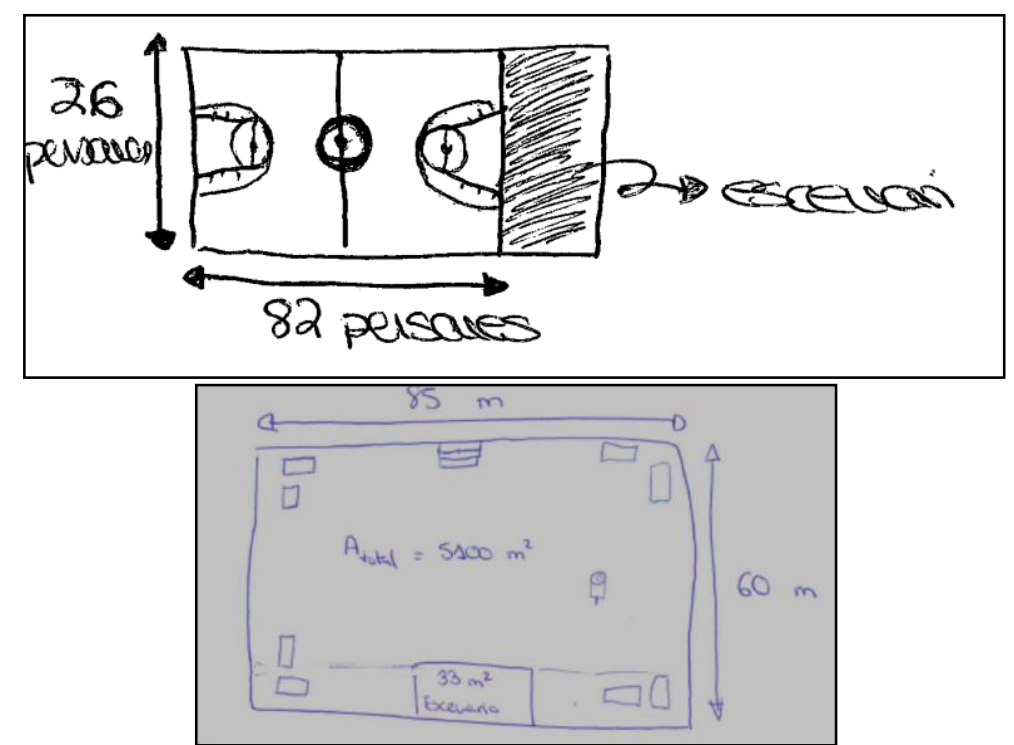

Figura 7 - Gráficos que muestran información recogida durante la actividad Fuente: producción alumnos de uno de los grupos experimentales del trabajo

Por otra parte, hemos observado otro uso de los gráficos para expresar procedimientos matemáticos que los alumnos no describen literalmente. En la Figura 8 se puede observar cómo un equipo de alumnos explica su proceso de medida del patio en un gráfico que acompaña al texto que se muestra en la Figura 3. Otro equipo de alumnos (del grupo E1) utiliza también un gráfico del mismo tipo en el problema A, en este caso lo hace para acompañar la explicación que les ayuda a obtener la expresión algebraica para la medida del área útil del patio que se muestra en la Figura 10. Este uso de los gráficos es particularmente interesante porque muestra que en ocasiones los alumnos hacen uso del lenguaje gráfico para expresar ideas que no son capaces de explicar con lenguaje literal o, en otros casos, parece que les ayuda a ilustrar ideas matemáticas abstractas (como una expresión general en forma algebraica).

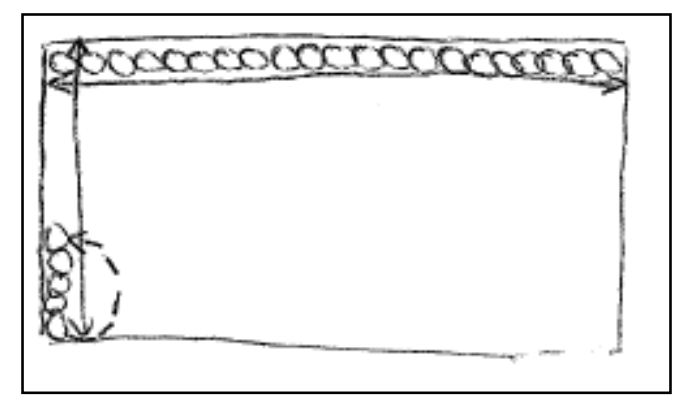

Figura 8 - Gráfico que muestra un proceso de recogida de datos (equipo E2) Fuente: producción alumnos de uno de los grupos experimentales del trabajo

En la Tabla 4 mostramos la distribución del uso de los dos tipos de gráficos en cada una de las tareas por parte de ambos grupos. 
Tabla 4 - Tipos de gráficos utilizados

\begin{tabular}{|l|l|l|l|l|}
\hline \multirow{2}{*}{ Problema } & \multicolumn{2}{|l|}{$\begin{array}{l}\text { Gráficos toma de } \\
\text { datos }\end{array}$} & \multicolumn{2}{|l|}{$\begin{array}{l}\text { Gráficos explicar } \\
\text { procedimientos }\end{array}$} \\
\cline { 2 - 5 } & E1 & E2 & E1 & E2 \\
\hline A & 2 & 3 & 1 & 1 \\
\hline B1 & 2 & 4 & 0 & 0 \\
\hline B2 & 4 & 1 & 2 & 0 \\
\hline B3 & 2 & 0 & 1 & 0 \\
\hline
\end{tabular}

Fuente: tabla realizada por los autores del trabajo

Para analizar los lenguajes escritos utilizados para expresar las resoluciones a los distintos problemas de la secuencia hemos establecido una clasificación que incluye los siguientes tipos de lenguaje: literal, aritmético, pre-algebraico y algebraico.

En muchos casos, los estudiantes desarrollan resoluciones que transmiten exclusivamente mediante un texto escrito en el que se encuentran conceptos matemáticos expresados de forma literal (véase la primera parte de la resolución mostrada en la Figura 10). En concreto, la resolución de 19 de los problemas recogidos se expresa de forma exclusivamente literal. Algunos equipos incorporan cálculos aritméticos a estas explicaciones, hasta recoger 26 resoluciones de problemas con lenguaje literal combinado con cálculos aritméticos. En la Tabla 5 mostramos el número de equipos de alumnos en cada equipo que han usado lenguaje literal exclusivo y aquellos que combinan lenguaje literal con operaciones aritméticas.

Tabla 5 - Uso del lenguaje literal

\begin{tabular}{|l|l|l|l|l|}
\hline \multirow{2}{*}{ Problema } & \multicolumn{2}{|l|}{$\begin{array}{l}\text { Literal } \\
\text { exclusivo }\end{array}$} & \multicolumn{2}{l|}{$\begin{array}{l}\text { Uso lenguaje literal y } \\
\text { aritmético }\end{array}$} \\
\cline { 2 - 5 } & E1 & E2 & E1 & E2 \\
\hline A & 1 & 1 & 4 & 5 \\
\hline B1 & 4 & 3 & 3 & 3 \\
\hline B2 & 3 & 2 & 2 & 3 \\
\hline B3 & 4 & 1 & 3 & 3 \\
\hline
\end{tabular}

Fuente: tabla realizada por los autores del trabajo

Respecto al uso de lenguajes (pre)-algebraicos encontramos dos usos diferenciados. Por un lado, observamos equipos que utilizan, además del lenguaje verbal, el lenguaje prealgebraico o, incluso, algebraico, para reproducir y manipular fórmulas conocidas (asociadas al cálculo de áreas) o realizar procedimientos (como cálculos de proporcionalidad utilizando la regla de tres). En estos casos es habitual que aparezcan, también, expresiones aritméticas que les permiten obtener soluciones numéricas aproximadas. Sin embargo, otros equipos hacen uso de lenguaje pre-algebraico para expresar ideas matemáticas propias en sus informes de trabajo. En efecto, hemos observado casos de uso de un tipo de lenguaje pre-algebraico para expresar relaciones matemáticas que se han construido en la resolución de estas 
actividades. La Figura 9 muestra los dos casos detectados en los que hemos observado este hecho, y que consideramos relevantes ya que muestran un intento por parte de los alumnos de ofrecer una expresión generalizable, introduciendo variables en una relación algebraica. Estos dos ejemplos pertenecen a equipos del grupo E1 y contrastan con el único ejemplo de lenguaje pre-algebraico observado por parte de un equipo del grupo E2, en el que los alumnos expresan una relación de área ya conocida.

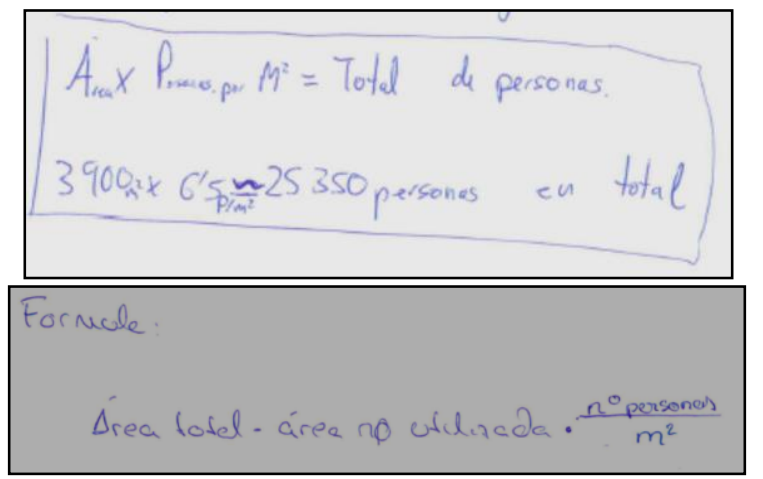

Figura 9 - Fragmentos de la resolución del problema A realizada por dos equipos del grupo E1 Fuente: producción alumnos de uno de los grupos experimentales del trabajo

Es importante destacar que ninguno de los equipos es capaz de formalizar completamente estas ideas expresándolas en lenguaje puramente algebraico, solo en algunos casos, se hace un uso combinado de ambos, véase la Figura 10.

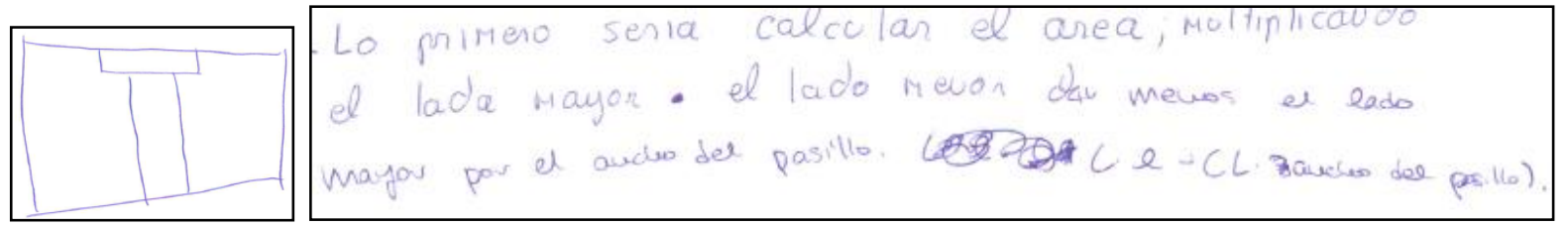

Figura 10 - Fragmentos de la resolución del problema A realizada por un equipo del grupo E1 Fuente: producción alumnos de uno de los grupos experimentales del trabajo

En la Tabla 6 se resumen los tipos de lenguaje detectados en las resoluciones analizadas, distinguiendo entre los dos usos de los lenguajes pre-algebraico y algebraico comentados previamente.

Tabla 6 - Tipos de lenguajes utilizados

\begin{tabular}{|l|l|l|l|l|l|l|}
\hline Problema & \multicolumn{2}{|l|}{ Aritmético } & \multicolumn{2}{l|}{$\begin{array}{l}\text { (Pre)- } \\
\text { algebraico } \\
\text { manipulación } \\
\text { de fórmulas }\end{array}$} & \multicolumn{2}{l|}{$\begin{array}{l}\text { (Pre)- } \\
\text { algebraico } \\
\text { ideas } \\
\text { propias }\end{array}$} \\
\cline { 2 - 8 } & E1 & E2 & E1 & E2 & E1 & E2 \\
\hline A & 4 & 5 & 2 & 1 & 3 & 0 \\
\hline B1 & 3 & 3 & 2 & 1 & 0 & 0 \\
\hline B2 & 1 & 4 & 2 & 1 & 0 & 0 \\
\hline B3 & 2 & 4 & 2 & 2 & 0 & 0 \\
\hline
\end{tabular}


Fuente: tabla realizada por los autores del trabajo

A continuación repasamos los hechos detectados más relevantes en el análisis de los tipos de lenguaje utilizados. Hemos observado que 5 de los 6 equipos de alumnos del grupo E2, sin experiencia previa en actividades de modelización, utilizan en el informe de solución del problema A un lenguaje que hemos clasificado como aritmético. En cambio, los equipos del grupo E1, para ese mismo problema utilizan lenguajes que incorporan otros elementos matemáticos, como el lenguaje algebraico. Es destacable, además, que tres de los equipos del grupo E1 obtienen en este problema fórmulas en lenguaje pre-algebraico que les permiten expresar ideas propias (véase Figura 10). Interpretamos esta diferencia en relación a la inexperiencia de los alumnos del grupo E2 en actividades de matemáticas en las que deben generar informes de respuesta elaborados. Además, al analizar las respuestas de los alumnos observamos que, en particular en el grupo E1, conforme avanzan en la resolución de problemas del mismo tipo los alumnos tienden a limitar sus resoluciones al lenguaje literal.

\section{Conclusiones}

En este estudio mostramos las diferencias identificadas en las producciones de dos grupos de estudiantes con distinto nivel de experiencia en modelización que se enfrentan a una secuencia de PEGC. Nuestro análisis se basa en caracterizar los modelos producidos durante la actividad a partir de identificar los conceptos, procedimientos y lenguajes utilizados por los alumnos. En concreto, este tipo de análisis nos permite identificar diferencias en los modelos matemáticos producidos durante el proceso de resolución de problemas, especialmente a nivel de los conceptos y lenguajes utilizados. Además, a partir del análisis realizado podemos observar que la resolución de los problemas de Fermi exige a los alumnos la elaboración de modelos que contienen un alto nivel de detalle para un tipo de problemas que pueden abordar a partir de sus propios conocimientos.

Por una parte, observamos que los alumnos con una experiencia previa en modelización utilizan conceptos matemáticos formales en sus resoluciones desde la primera tarea. Pero el aspecto diferenciador más relevante es una mayor complejidad en los modelos producidos a base de introducir variaciones en los mismos para adaptarse a la naturaleza de los problemas trabajados. Estas variaciones son adaptaciones del modelo generado para resolver el problema A a las características de las situaciones estudiadas en los problemas B de la secuencia. En particular, la distinción de zonas hábiles o inhábiles en una superficie es el 
elemento de modificación de los modelos más utilizado. Entendemos que este tipo de modificación de los modelos es más simple que la diferenciación de zonas en las que aplicar valores distintos de densidad, que es el otro tipo de elemento de complejidad que hemos identificado.

Para los procedimientos utilizados, solo hemos encontrado como elemento diferenciador que dos de los equipos del grupo sin experiencia previa (E2) utilizan procesos de medida no formales para el problema $\mathrm{A}$. Si nos centramos en los lenguajes utilizados, hemos observado que los alumnos del grupo E1 consiguen elaborar expresiones algebraicas que son generalizables, cuando los alumnos del grupo E2 se limitan a proponer expresiones que únicamente soportan un cálculo puntual, utilizando esencialmente un lenguaje aritmético. Entendemos que estos alumnos del grupo E1 han tomado consciencia de la necesidad de generar contenido matemático adaptable a otras situaciones y han adquirido e incorporado esta práctica de trabajo. Por otra parte, y en referencia a los tipos de lenguaje observados, hemos encontrado diferencias en el uso de gráficos para expresar procesos matemáticos que los alumnos no saben describir utilizando lenguaje escrito.

Si nos centramos en la potencialidad del tipo de análisis utilizado, observamos que nos ha permitido caracterizar las producciones de los alumnos con un alto nivel de detalle, suficiente para observar diferencias entre las producciones de los diferentes grupos, pero también para observar diferencias en los modelos producidos para los distintos problemas. Una muestra es el mayor el uso de la iteración de una unidad para resolver el problema B3, cuando la densidad de población es el concepto que acaba siendo mayoritario en el resto de problemas B - en especial después de la puesta en común realizada.

Por otra parte, el análisis realizado nos permite observar, también, la evolución presente en las producciones de los alumnos sin experiencia previa mientras se desarrolla la actividad. En particular, hemos observado que los alumnos intentan formalizar matemáticamente algunas ideas genuinas pero, al fijarnos en detalle en los lenguajes utilizados, vemos que no alcanzan el nivel de abstracción que muestran los alumnos con experiencia. Cabe pensar que los lenguajes utilizados evolucionan conforme los alumnos resuelven nuevos problemas de este tipo, ya que este tipo de problemas les permite ver la potencia del lenguaje algebraico a la hora de construir modelos generalizables y, por tanto, reutilizables en otros contextos.

Desde el punto de vista del análisis de actividades de modelización, en Gallart, Ferrando y García-Raffi (2015a) se observa que el ciclo de modelización propuesto por Blum 
y Leiss (2007) proporciona una herramienta útil para evaluar la actuación de los estudiantes al enfrentarse a una tarea de modelización, identificando las competencias que se activan en cada una de las fases durante el proceso de resolución. En esta línea, entendemos que el tipo de análisis que aportamos en este estudio - siguiendo la definición de modelo matemático de Lesh y Harel (2003) - complementa a la herramienta anterior. Consideramos que la consideración de conceptos, procedimientos y lenguajes utilizados en la descripción de modelos matemáticos puede permitir establecer una herramienta de análisis que sea útil tanto para los investigadores que analicen actividades de modelización como para los profesores en el aula.

Consideramos que este tipo de análisis puede adaptarse y ser útil en otro tipo de actividades de modelización y que puede establecer un punto de partida para elaborar pautas de evaluación de modelos matemáticos producidos por los alumnos o para preparar la anticipación de la actividad por parte del profesorado y seguiría la línea de dotar al profesor de herramientas con las que llevar adelante con éxito experiencias de aula basadas en la modelización matemática (GALLART; FERRANDO; GARCÍA-RAFFI, 2015b).

\section{Referencias}

ALBARRACÍN, L.; GORGORIÓ, N. Problemas de estimación de grandes cantidades: modelización e influencia del contexto. Revista Latinoamericana de Investigación en Matemática Educativa, Méjico, v. 16, n. 3, p. 289-315, 2013.

ALBARRACÍN, L.; GORGORIÓ, N. Devising a plan to solve Fermi problems involving large numbers. Educational Studies in Mathematics, Dordrecht, v. 86, n. 1, p. 79-96, 2014.

ÄRLEBÄCK, J. B. On the use of realistic Fermi problems for introducing mathematical modelling in school. The Montana Mathematics Enthusiast, Montana, v. 6, n. 3, p. 331-364, 2009.

ÄRLEBÄCK, J. B. Exploring the solving process of groups solving realistic fermi problem from the perspective of the anthropolgical theory of didactics. In: CONGRESS OF THE EUROPEAN SOCIETY FOR RESEARCH IN MATHEMATICS EDUCATION, 7., 2011, Rzeszów. Proceedings of CERME 7, Rzeszów: ERME, 2011. p. 1010-1019.

BLUM, W. ICMI (2002) Study 14: applications and modelling in mathematics education: discussion document. Educational Studies in Mathematics, Dordrecht, v. 51, n. 1-2, p. 149-171, 2012.

BLUM, W.; LEISS, D. Filling Up: the problem of independence-preserving teacher interventions in lessons with demanding modelling tasks. In: CONGRESS OF THE EUROPEAN SOCIETY FOR RESEARCH IN MATHEMATICS EDUCATION, 4., Sant Feliu de Guixols. Proceedings of CERME 4, Sant Feliu de Guixols: ERME, 2007. p. 1623-1633.

BORROMEO-FERRI, R. Theoretical and empirical differentiations of phases in the modelling process. ZDM, Heidelberg, v. 38, n. 2, p. 86-95, 2006. 
CARLSON, J. E. Fermi problems on gasoline consumption. The Physics Teacher, New York, v. 35, p. 308-309, 1997.

EFTHIMIOU, C. J.; LLEWELLYN, R. A. Cinema, Fermi problems and general education. Physics Education, London, v. 42, n. 3, p. 253-261, 2007.

GALLART C.; FERRANDO I.; GARCÍA-RAFFI, L. M. Análisis competencial de una tarea de modelización abierta. Números, La Laguna, v. 88, p. 93-103, 2015 a.

GALLART C.; FERRANDO I.; GARCÍA-RAFFI, L. M. El profesor ante la actividad modelizadora en el aula de secundaria. SUMA, Madrid, v. 79, p. 9-16, 2015 b.

GARCÍA-NAVARRO, J. M. Problemas de Fermi. Suposición, estimación y aproximación. Epsilon, Sevilla, v. 30, n. 2, p. 57-68, 2013.

HIEBERT, J.; LEFEVRE, P. Conceptual and procedural knowledge in mathematics: an introductory analysis. In: HIEBERT, J. (Ed.) Conceptual and Procedural Knowledge: the Case of Mathematics. Hillsdale, NJ, US: Lawrence Erlbaum Associates, 1986. p. 1-27

HILDRETH, D. J. The use of strategies in estimating measurements. The Arithmetic Teacher, Washington, v. 30, n. 5, p. 50-54, 1983.

LESH, R.; HAREL, G. Problem solving, modeling, and local conceptual development. Mathematical Thinking and Learning, Mahwah, v. 5, n. 2, p. 157-189, 2003.

LESH, R.; HOOVER, M.; HOLE, B.; KELLY, A.; POST, T. Principles for developing thoughtrevealing activities for students and teachers. In: KELLY, A. E.; LESH, R. Handbook of Research Design in Mathematics and Science Education. Mahwah: Ed. Erlbaum, 2000. p. 591-645.

LESTER, F. K. Musings about mathematical problem-solving research: 1970-1994. Journal for Research in Mathematics Education, Reston, v. 25, n. 6, p. 660-675, 1994.

PETER-KOOP, A. Teaching and understanding mathematical modelling through Fermi-problem. In: CLARKE, B.; GREVHOLM, B.; MILLMAN, R. Tasks in Primary Mathematics Teacher Education. Dordrecht: Ed. Springer, 2009. p. 131-146.

REAL DECRETO 1105/2014, de 26 de diciembre, por el que se establece el currículo básico de la Educación Secundaria Obligatoria y del Bachillerato. Boletín Oficial del Estado, núm. 3, Sec. I., 3 de enero de 2015, p. 169-546.

SEGOVIA, I.; CASTRO, e.; Castro, E; Rico, L. Estimación en Cálculo y Medida. Madrid: Ed. Síntesis, 1989. p. 1-208.

SCHOENFELD, A. H. Problem solving in the United States, 1970-2008: research and theory, practice and politics. ZDM, Heidelberg, v. 39, n. 5-6, p. 537-551, 2007.

SILVA CAMPOS, I. da; LOIOLA ARAÚJO, J. de. Envolvimento dos alunos em atividades de modelagem matemática: relação com o saber e possibilidades de ação. Boletim de Educação Matemática, Rio Claro, v. 29, n. 51, p. 167-182, 2015.

SRIRAMAN, B.; KNOTT, L. The mathematics of estimation: possibilities for interdisciplinary pedagogy and social consciousness. Interchange, Dordrecht, v. 40, n. 2, p. 205-223, 2009. 
WESSELS, H. M. Levels of mathematical creativity in model-eliciting activities. Journal of Mathematical Modelling and Application, Blumenau, v. 1, n. 9, p. 22-40, 2014.

Submetido em Junho de 2016. Aprovado em Setembro de 2016. 